PSS PROCEEDINGS

\title{
Measurements of |Vus| and Searches for Violation of Lepton Universality and CPT in Tau Decays at BABAR
}

\author{
Alberto Lusiani ${ }^{* \dagger}$ \\ Scuola Normale Superiore and INFN - Pisa \\ E-mail: alberto.lusiani@pi.infn.it
}

Using data collected with the BABAR detector at PEP-II at SLAC, we report on several tau lepton measurements that are used to determine the modulus of the Cabibbo-Kobayashi-Maskawa matrix element $\left|V_{u s}\right|$, and to check the Standard Model predictions of lepton universality and $C P T$ conservation.

35th International Conference of High Energy Physics

July 22-28, 2010

Paris, France

* Speaker.

${ }^{\dagger}$ Representing the BABAR collaboration 


\section{One-prong tau decay branching fractions}

A recent publication by the BABAR collaboration reports on precise measurements of the 1prong tau decay branching fractions [1], using $467 \mathrm{fb}^{-1}$ of $e^{+} e^{-}$annihilation data at the $\Upsilon(4 S)$ peak, produced at PEP-II (SLAC).

At the B-factories, precision tau branching fractions measurements are limited by systematic uncertainties that are at present significantly higher than the ones that were obtained at the LEP experiments[2]. Some of the largest systematic uncertainties, such as the luminosity related ones, are common to different channels, and cancel out when measuring ratios of branching fractions. To best exploit systematics cancellations, the BABAR collaboration has measured in a single analysis four 1-prong tau branching fractions: $\mathscr{B}\left(\tau^{-} \rightarrow e^{-} \bar{v}_{e} v_{\tau}\right), \mathscr{B}\left(\tau^{-} \rightarrow \mu^{-} \bar{v}_{\mu} v_{\tau}\right), \mathscr{B}\left(\tau^{-} \rightarrow \pi^{-} v_{\tau}\right)$ and $\mathscr{B}\left(\tau^{-} \rightarrow K^{-} v_{\tau}\right)$.

At the $\Upsilon(4 S)$ center-of-mass (CM) energy, when reconstructing tracks in the CM frame, both tau leptons are boosted and their decay products are well separated in two opposite hemispheres, which can be conveniently defined using the reconstructed event thrust axis. For all channels, signal candidates are selected by requiring an event topology consisting in one prong in the signal side against three prongs in the tag side. Tight particle identification is used in order to suppress cross-feed background between the measured modes, achieving clean selections. Backgrounds, cross-feeds, and the performance of particle identifications requirements are carefully estimated using a variety of Monte Carlo and data control samples.

The resulting measurements determine ratios of branching fractions where the limiting systematics are determined by uncertainties on the particle identification signal efficiencies and background suppressions, and on uncertainties on background contaminations from channels other than the ones that were simultaneously analysed. Systematics from luminosity, from the $e^{+} e^{-} \rightarrow \tau^{+} \tau^{-}$ cross-section and from the branching fraction and efficiency of modes on the remaining tau lepton into a 3-prong final state all cancel at first order. Furthermore, using the electron branching fraction world average [3] as normalization, the BABAR analysis determines precise muon, pion and kaon branching fractions, which are comparable to the 2008 world averages [3] for $\mathscr{B}\left(\tau^{-} \rightarrow \mu^{-} \bar{v}_{\mu} v_{\tau}\right)$ and $\mathscr{B}\left(\tau^{-} \rightarrow \pi^{-} v_{\tau}\right)$ and about four times more precise for $\mathscr{B}\left(\tau^{-} \rightarrow K^{-} v_{\tau}\right)$. The measured ratios of branching fractions are $\mathscr{B}\left(\tau^{-} \rightarrow \mu^{-} \bar{v}_{\mu} \nu_{\tau}\right) / \mathscr{B}\left(\tau^{-} \rightarrow e^{-} \bar{v}_{e} \nu_{\tau}\right)=0.9796 \pm 0.0016 \pm 0.0036$, $\mathscr{B}\left(\tau^{-} \rightarrow \pi^{-} v_{\tau}\right) / \mathscr{B}\left(\tau^{-} \rightarrow e^{-} \bar{v}_{e} v_{\tau}\right)=0.5945 \pm 0.0014 \pm 0.0061, \mathscr{B}\left(\tau^{-} \rightarrow K^{-} v_{\tau}\right) / \mathscr{B}\left(\tau^{-} \rightarrow\right.$ $\left.e^{-} \bar{v}_{e} v_{\tau}\right)=0.03882 \pm 0.00032 \pm 0.00057$. The paper also quotes the full covariance matrix.

\section{Improvements on Lepton Universality Tests}

The above mentioned BABAR publication [1] uses the precise ratios of branching fractions to test lepton universality for the weak charged current couplings. A test of $\mu-e$ universality is obtained from the ratio $\mathscr{B}\left(\tau^{-} \rightarrow \mu^{-} \bar{v}_{\mu} v_{\tau}\right) / \mathscr{B}\left(\tau^{-} \rightarrow e^{-} \bar{v}_{e} v_{\tau}\right),\left(g_{\mu} / g_{e}\right)^{2}=1.0036 \pm 0.0020$, which in combination with the world average [3] yields $\left(g_{\mu} / g_{e}\right)_{\tau}^{2}=1.0018 \pm 0.0014$, which is consistent with the Standard Model (SM) prediction and with the same universality test that is obtained from pion decays, $\left(g_{\mu} / g_{e}\right)_{\pi}^{2}=1.0021 \pm 0.0015[3,4]$. Using the precise ratios $\mathscr{B}\left(\tau \rightarrow h v_{\tau}\right) / \mathscr{B}\left(\tau^{-} \rightarrow\right.$ $\left.\mu^{-} \bar{v}_{\mu} v_{\tau}\right)$ with $h=\pi, K$ and other well known quantities such as the tau, pion and kaon masses and lifetimes [3], muon-tau universality tests are determined using respectively the pion channel, the 
kaon channel, and both of them in combination: $\left(g_{\tau} / g_{\mu}\right)_{\pi}^{2}=0.986 \pm 0.006,\left(g_{\tau} / g_{\mu}\right)_{K}^{2}=0.983 \pm$ $0.009,\left(g_{\tau} / g_{\mu}\right)_{h}^{2}=0.985 \pm 0.005$. The last value is $2.8 \sigma$ below the SM expectation and within $2 \sigma$ of the world average [3].

\section{Measurement of $\mathscr{B}\left(\tau^{-} \rightarrow K_{S}^{0} \pi^{-} \pi^{0} v_{\tau}\right)$}

The BABAR collaboration has produced a preliminary measurement of $\mathscr{B}\left(\tau^{-} \rightarrow K_{S}^{0} \pi^{-} \pi^{0} v_{\tau}\right)$ [5]. Candidate events are required to have, in the CM system, one hemisphere containing one single prong identified as an electron or muon, and the opposite hemisphere containing a $\pi^{-}$, a $\pi^{+} \pi^{-}$ pair compatible with a $K_{S}^{0}$ decay, and a reconstructed $\pi^{0}$ from two detected photons. This channel is affected by large backgrounds from the processes where the signal tau decays into $K_{S}^{0} \pi^{-} v_{\tau}$ and $K_{S}^{0} \pi^{-} K_{L}^{0} v_{\tau}$, however it is possible to select candidates with relative high purity by requiring that the $\pi^{0}$ energy in the CM system is larger than $1.2 \mathrm{GeV}$. In so doing, one selects events in a restricted region of the phase space, therefore it is crucial to have an accurate simulation of the data in order to precisely estimate the efficiency of the selection. Therefore $B A B A R$ has studied the invariant mass distributions of all couples of the final state particles $\left(K_{S}^{0}, \pi^{-}, \pi^{0}\right)$ in the pure selected sample of candidates and has tuned the Monte Carlo generator to the data. The measured branching fraction is $\mathscr{B}\left(\tau^{-} \rightarrow \bar{K}^{0} \pi^{-} \pi^{0} v_{\tau}\right)=0.342 \pm 0.006$ (stat) \pm 0.015 (syst).

\section{Determination of $\left|V_{u s}\right|$}

Several experimental measurements can be combined to determine the modulus of the CabibboKobayashi-Maskawa matrix element $\left|V_{u s}\right|$, as summarized by Table 1 . The most precise determinations come from precise kaon measurements and are presently limited by QCD theory systematic uncertainties.

The above mentioned BABAR publication [1] reports two $\left|V_{u s}\right|$ determinations directly obtained from the measured branching fractions, one using the ratio of kaon to pion branching fractions, i.e. the "tau $K / \pi$ " method in Table $1\left(\left|V_{u s}\right|=0.2255 \pm 0.0024\right.$, using the $\left|V_{u d}\right|$ measurement in Ref. [11]), and a second one using the " $\tau \rightarrow K v$ exclusive" method, $\left|V_{u s}\right|=0.2193 \pm 0.0032$.

The ratio $\left|V_{u s}\right| /\left|V_{u d}\right|$ can be determined from the ratio of the decay widths of the tau to strange and non-strange hadronic final states, up to a $S U(3)_{\text {flavor }}$ breaking correction determined by the $s$ quark mass and QCD [9]. Using the ratios $R_{\tau, s}=\Gamma(\tau \rightarrow$ strange hadronic final states $/ \Gamma(\tau \rightarrow e v \bar{v})$ and $R_{\tau, V+A}=\Gamma(\tau \rightarrow$ non-strange hadronic final states $/ \Gamma(\tau \rightarrow e v \bar{v})$, the following relation holds, according to the " $\tau \rightarrow X_{S} v$ inclusive" method in Table 1:

$$
\left|V_{u s}\right|^{2}=R_{\tau, s} /\left(\frac{R_{\tau, V+A}}{\left|V_{u d}\right|^{2}}-\delta R_{\tau, \text { SU3 breaking }}\right)
$$

To determine $R_{\tau, s}$ and $R_{\tau, V+A}$, we use all tau branching fraction measurements reported in Ref [3], including the 2009 partial update for the 2010 edition (PDG2009) and all the available more recent measurements from the $\mathrm{B}$-factories Belle and $B A B A R$, including preliminary ones, as detailed in the HFAG 2010 update [12]. We average all measurements, taking into account correlations for the B-factories measurements, in a single global unconstrained fit, with a minimum $\chi^{2}$ fit. Since 
Measurements of $\mid$ Vus $\mid$ and Searches for Violation of Lepton Universality and CPT in Tau Decays at BABAR Alberto Lusiani

\begin{tabular}{|c|c|c|c|}
\hline method & illustrative formula & $\Delta_{\mathrm{th}}$ & Ref. \\
\hline$K_{\ell 3}$ & $=\left[\left|V_{u s}\right| f_{+}(0)\right]^{2} \times\langle$ well known constants $\rangle$ & $0.58 \%$ & {$[6]$} \\
\hline$K_{\ell 2}(K / \pi)$ & $\frac{\Gamma\left(K_{\ell 2}\right)}{\Gamma\left(\pi_{\ell 2}\right)} \quad=\left|\frac{V_{u s}}{V_{u d}}\right|^{2} \frac{f_{K}^{2}}{f_{\pi}^{2}} \times\langle$ well known constants $\rangle$ & $0.50 \%$ & [7] \\
\hline $\operatorname{tau} K / \pi$ & $\frac{\Gamma(\tau \rightarrow K v)}{\Gamma(\tau \rightarrow \pi v)}=\left|\frac{V_{u s}}{V_{u d}}\right|^{2} \frac{f_{K}^{2}}{f_{\pi}^{2}} \times\langle$ well known constants $\rangle$ & $0.50 \%$ & [7] \\
\hline$\tau \rightarrow K \nu$ exclusive & $\mathscr{B}\left(\tau^{-} \rightarrow K^{-} v_{\tau}\right)=\frac{G_{F}^{2} f_{K}^{2}\left|V_{u s}\right|^{2} m_{\tau}^{3} \tau_{\tau}}{16 \pi \hbar}\left(1-\frac{m_{K}^{2}}{m_{\tau}^{2}}\right)^{2} S_{E W}$ & $1.27 \%$ & {$[8]$} \\
\hline$\tau \rightarrow X_{S} \nu$ inclusive & $=\frac{R_{\tau, V+A}}{\left|V_{u d}\right|^{2}}-\delta R_{\tau, \mathrm{SU} 3 \text { breaking }}$ & $0.23 \%-0.47 \%$ & {$[9,10]$} \\
\hline unitarity & $=1-\left|V_{u d}\right|^{2}-\left|V_{u b}\right|^{2}$ & & \\
\hline
\end{tabular}

Table 1: Combinations of experimental measurements to compute $\left|V_{u s}\right|: \Delta_{\text {th }}$ identifies the theoretical uncertainties due to QCD as determined by the reference(s) on the right. $R_{\tau, s}=\Gamma(\tau \rightarrow$ strange hadronic final states $) / \Gamma(\tau \rightarrow e v \bar{v}), R_{\tau, V+A}=\Gamma(\tau \rightarrow$ non-strange hadronic final states $) / \Gamma(\tau \rightarrow$ $e v \bar{v})$.

there is a large discrepancy between the measurements of $\tau \rightarrow K^{-} K^{+} K^{-} v$ by BABAR [13] and Belle [14], a PDG-style error scale factor (5.44) has been used.

We define the branching fraction of the tau to a strange hadronic final state as described in Table 2 (which is slightly different from the not-yet-finalized HFAG-tau definition [12]).

In the literature, e.g. Refs. $[9,10], R_{\tau, s}$ is obtained by summing all strange decay modes, while $R_{\tau, V+A}$ is obtained by difference using unitarity for sum of the tau branching fractions, i.e. $R_{\tau, V+A}=R_{\tau, h}-R_{\tau, s}$ with $R_{\tau, h}=\left(1-B_{e}-B_{\mu}\right) / B_{e}$, where $B_{e}, B_{\mu}$ are the leptonic tau branching fractions computed assuming SM lepton universality and using also the tau lifetime world average [15]. This method implicitly assigns all possibly not-yet-measured or neglected hadronic tau branching fractions to $R_{\tau, V+A}$. To prevent that, we rather determine $R_{\tau, V+A}$ by summing up all non-strange modes. We obtain $\left|V_{u s}\right|=0.2166 \pm 0.0023$, which is $3.57 \sigma$ away from $\left|V_{u s}\right|=0.2255 \pm 0.0010$ obtained from CKM unitarity using Ref. [11]. We still use the improved $B_{e}$ assuming SM lepton universality to compute $R_{\tau, V+A}$ and $R_{\tau, s}$. Figure 1 reports comparison with other $\left|V_{u s}\right|$ determinations. When using the literature definition for $R_{\tau, V+A}$, there are no significant changes: $\left|V_{u s}\right|=0.2163 \pm 0.0023,3.66 \sigma$ away from unitarity. By inflating measurement errors in the global fit with a procedure similar to the one used by PDG for error scale factors, we obtain $\left|V_{u s}\right|=0.2166 \pm 0.0025$, corresponding to a slightly reduced discrepancy of $3.29 \sigma$.

\section{Test of CPT conservation from tau mass measurements}

The BABAR collaboration has recently published a tau mass measurement [16] obtained by fitting the end-point of the reconstructed minimum tau mass for $\tau \rightarrow 3 \pi v$ candidates. The measurement is robust against background contamination, is statistically precise but is ultimately systemat- 


\begin{tabular}{ll}
\hline hadronic system in $\tau \rightarrow X_{s} v$ & fitted branching fraction \\
\hline$K^{-}$ & $0.696 \pm 0.010$ \\
$K^{-} \pi^{0}$ & $0.431 \pm 0.015$ \\
$\bar{K}^{0} \pi^{-}$ & $0.827 \pm 0.018$ \\
$K^{-} \pi^{0} \pi^{0}$ & $0.060 \pm 0.022$ \\
$\bar{K}^{0} \pi^{-} \pi^{0}$ & $0.348 \pm 0.015$ \\
$\bar{K}^{0} \pi^{-} \pi^{0} \pi^{0}$ & $0.023 \pm 0.023$ \\
$K^{-} \pi^{-} \pi^{+}$ & $0.293 \pm 0.007$ \\
$K^{-} \pi^{-} \pi^{+} \pi^{0}$ & $0.077 \pm 0.012$ \\
$K^{-} \pi^{0} \pi^{0} \pi^{0}$ & $0.040 \pm 0.022$ \\
$\bar{K}^{0} h^{-} h^{+} h^{-}$ & $0.023 \pm 0.020$ \\
$K^{-} \eta$ & $0.016 \pm 0.001$ \\
$K^{-} \pi^{0} \eta$ & $0.0048 \pm 0.0012$ \\
$\bar{K}^{0} \pi^{-} \eta$ & $0.0094 \pm 0.0015$ \\
$K^{-} K^{+} K^{-}$ & $0.0023 \pm 0.0008(S=5.44)$ \\
$K^{-} K^{0} \bar{K}^{0}$ & from $K^{-} K^{+} K^{-} \cdot \frac{\phi \rightarrow K^{0} \bar{K}^{0}}{\phi \rightarrow K^{+} K^{-}}$ \\
\hline$\tau \rightarrow X_{s} v$ & $0.0016 \pm 0.0006(S=5.44)$ \\
\hline
\end{tabular}

Table 2: List of modes used to compute $\mathscr{B}\left(\tau \rightarrow X_{s} v\right)$. Since the $\tau^{-} \rightarrow K^{-} K^{+} K^{-} v_{\tau}$ mode appears to be saturated by the $\tau^{-} \rightarrow K^{-} \phi v_{\tau}$ mode, we use the $\tau^{-} \rightarrow K^{-} K^{+} K^{-} v$ measurement to estimate the $\tau^{-} \rightarrow$ $K^{-} K^{0} K^{0} v$ mode using the $\phi$ branching fractions to $K^{+} K^{-}$and $K^{0} K^{0}$. An error scale factor $S=5.44$ has been used to inflate the errors of the inconsistent BABAR and Belle measurements for $\tau \rightarrow K^{-} K^{+} K^{-} v$.

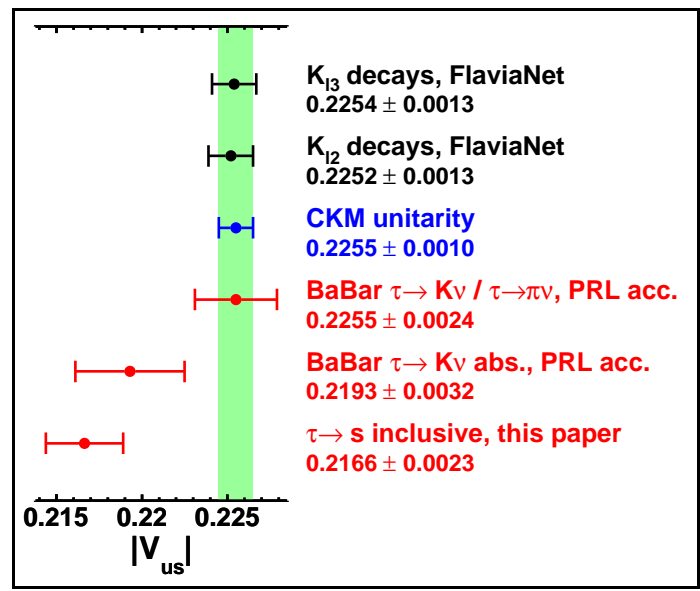

Figure 1: Determinations of $\left|V_{u s}\right|$. The FlaviaNet numbers come from Ref. [7], the sources of the other data are mentioned in the text.

ically limited by the calibration of the reconstructed momentum scale, remaining less competitive with respect to measurements at the $\tau^{+} \tau^{-}$production threshold that use resonant beam depolarization to measure the beam energy. However, unlike threshold experiments, BABAR can measure the mass of positive and negative tau leptons separately, providing a competitive test of $C P T$ conservation. The tau mass is measured to be $m_{\tau}=1776.68 \pm 0.12$ (stat) \pm 0.41 (syst) $\mathrm{MeV}$ and the mass difference is $\left(m_{\tau+}-m_{\tau-}\right) / m_{\text {average }}=-3.4 \pm 1.3$ (stat.) \pm 0.3 (syst. $) \cdot 10^{-4}$. There is no evidence of $C P T$ violation as in the similar published measurement by Belle [17]. 


\section{References}

[1] BaBar Collaboration, B. Aubert et. al., Measurements of Charged Current Lepton Universality and $\left|V_{u s}\right|$ using Tau Lepton Decays to $e^{-} \bar{v}_{e} v_{\tau}, \mu^{-} \bar{v}_{\mu} v_{\tau}, \pi^{-} v_{\tau}$, and $K^{-} v_{\tau}$, Phys. Rev. Lett. 105 (2010) 051602, [arXiv:0912.0242].

[2] A. Lusiani, Experimental Review on Lepton Universality and Lepton Flavour Violation tests at the B-factories, PoS KAON (2008) 054, [arXiv: 0709.1599$].$

[3] Particle Data Group Collaboration, C. Amsler et. al., Review of particle physics, Phys. Lett. B667 (2008) 1.

[4] V. Cirigliano and I. Rosell, $\pi / K \rightarrow e v$ branching ratios to $O\left(e^{2} p^{4}\right)$ in Chiral Perturbation Theory, JHEP 10 (2007) 005, [arXiv: 0707.4464 ].

[5] BaBar Collaboration, S. Paramesvaran, Selected topics in tau physics from BaBar, arXiv:0910.2884.

[6] P. A. Boyle et. al., K->pi form factors with reduced model dependence, arXiv: 1004.0886.

[7] M. Antonelli et. al., An evaluation of $\mid$ Vus $\mid$ and precise tests of the Standard Model from world data on leptonic and semileptonic kaon decays, Eur. Phys. J. C69 (2010) 399-424, [arXiv: 1005 . 2323].

[8] HPQCD Collaboration, E. Follana, C. T. H. Davies, G. P. Lepage, and J. Shigemitsu, High Precision determination of the $\pi, K, D$ and $D_{s}$ decay constants from lattice $Q C D$, Phys. Rev. Lett. 100 (2008) 062002, [arXiv:0706.1726].

[9] E. Gamiz, M. Jamin, A. Pich, J. Prades, and F. Schwab, $|V(u s)|$ and $m(s)$ from hadronic tau decays, Nucl. Phys. Proc. Suppl. 169 (2007) 85-89, [hep-ph / 0612154$].$

[10] E. Gamiz, M. Jamin, A. Pich, J. Prades, and F. Schwab, Theoretical progress on the $V_{u}$ s determination from tau decays, PoS KAON (2008) 008, [arXiv : 0709 . 0282].

[11] J. C. Hardy and I. S. Towner, Superallowed $0+$ to $0+$ nuclear beta decays: A new survey with precision tests of the conserved vector current hypothesis and the standard model, Phys. Rev. $\mathbf{C 7 9}$ (2009) 055502, [arXiv: 0812.1202].

[12] The Heavy Flavor Averaging Group, Averages of b-hadron, c-hadron, and tau-lepton Properties, arXiv:1010.1589.

[13] BaBar Collaboration, B. Aubert et. al., Exclusive branching fraction measurements of semileptonic tau decays into three charged hadrons, $\tau^{-} \rightarrow \phi \pi^{-} \nu_{\tau}$ and $\tau^{-} \rightarrow \phi K^{-} v_{\tau}$, Phys. Rev. Lett. 100 (2008) 011801, [arXiv:0707.2981].

[14] Belle Collaboration, M. J. Lee et. al., Measurement of the branching fractions and the invariant mass distributions for $\tau^{-} \rightarrow h^{-} h^{+} h^{-} v_{\tau}$ decays, Phys. Rev. D81 (2010) 113007, [arXiv: 1001.0083 ].

[15] M. Davier, A. Hocker, and Z. Zhang, The physics of hadronic tau decays, Rev. Mod. Phys. 78 (2006) 1043-1109, [hep-ph/0507078].

[16] BaBar Collaboration, B. Aubert et. al., Measurements of the $\tau$ Mass and Mass Difference of the $\tau^{+}$ and $\tau^{-}$at BaBar, Phys. Rev. D80 (2009) 092005, [arXiv: 0909.3562 ].

[17] Belle Collaboration, K. Abe et. al., Measurement of the mass of the tau-lepton and an upper limit on the mass difference between tau+ and tau-, Phys. Rev. Lett. 99 (2007) 011801, [hep-ex/ 0608046 ]. 